\title{
A Case of Recurrent Kikuchi-Fujimoto Disease
}

\author{
Majid Reza Erfanian Taghvaei ${ }^{1}$; Maryam Mirzaie ${ }^{1, *}$; Ali Parsa $^{2}$; Taghi Ghiasi Moghadam ${ }^{3}$ \\ ${ }^{1}$ Department of Community Medicine, Mashhad University of Medical Sciences, Mashhad, IR Iran \\ ${ }^{2}$ Orthopedic Research Center, Mashhad University of Medical Sciences, Mashhad, IR Iran \\ 3 Department of Pathology, Mashhad University of Medical Sciences, Mashhad, IR Iran \\ ${ }^{*}$ Corresponding author: Maryam Mirzaie, Department of Community Medicine, Mashhad University of Medical Sciences, Mashhad, IR Iran. Tel: +98-5118828888, Fax: +98-5118828560, \\ E-mail: Maryam_Mirzaie@hotmail.com
}

Received: November 27, 2014; Revised: March 19, 2015; Accepted: April 10, 2015

\begin{abstract}
Introduction: Kikuchi-Fujimoto Disease (KFD) is a benign, self-limited, inflammatory disorder, first reported in Japan. This condition is more prevalent among women and typically occurs in the third decade of life. It normally manifests as persistent, isolated cervical adenopathy with a recurrence rate of $3 \%$. The identification of this condition is of high significance, given the risk of misdiagnosis with other disorders such as malignant lymphoma and extensive necrosis.

Case Presentation: The patient was a 32-year-old female diagnosed with Kikuchi-Fujimoto disease via neck lymph node biopsy in August 2006 in the city of Mashhad, Iran. The disease regressed with proper follow-up, although after eight years the patient was readmitted to the hospital with severe weight loss, high fever, and uncommon symptoms of generalized adenopathy in cervical, axillary and inguinal regions.

Conclusions: Although KFD is an uncommon condition, it should be featured in the list of differential diagnoses of tender lymphadenopathy, especially lymphadenopathy localized to the cervical region. We reported a case of KFD with a prolonged relapse of eight years. Full recovery with a good response to corticosteroid regimen was achieved after the recurrence.
\end{abstract}

Keywords: Kikuchi-Fujimoto Disease; Recurrent; Cervical Lymphadenopathy

\section{Introduction}

Kikuchi-Fujimoto Disease (KFD), also known as histiocytic necrotising lymphadenitis (HNL), was first reported in Japan in 1972. Kikuchi and Fujimoto simultaneously but independently described the histological and clinical features of this inflammatory disease of lymph nodes and termed it as "subacute cervical necrotizing lymphadenitis". The disease was later called HNL or Kikuchi's disease (1). This disease occurs in all races, although the majority of cases have been reported among Asians (2). The etiology of KFD is unknown, although some believe that viral agents are the main causes of this condition. KFD usually occurs as a persistent, isolated adenopathy and often appears in the neck area. This disease has been reported in different age groups ( 9 - 40 years old) and is more prevalent among women (3). Definite diagnosis of KFD is dependent on the biopsy and histology of lymph nodes $(4,5)$. This disease usually resolves within 1 - 3 months and recurrence and death are quite rare (6).

\section{Case Presentation}

The patient was a 32-year-old female, a native of Sistan and Baluchestan province, Iran, with complaints of afternoon fever $\left(38^{\circ} \mathrm{C}\right)$, night sweats, severe weight loss and anorexia. She was referred to the division of infectious diseases at Shahid Hashemi Nejad hospital in Mashhad,
Iran, in August 2006. A month before the admission, she had received three different doses of ceftriaxone and acetaminophen for her high fever; given her non-responsiveness to the treatment, she was readmitted to the hospital. The patient was previously healthy and newly married, with no prior history of diseases or drug consumption. She was lethargic, though hemodynamically stable according to physical examinations, and her body mass index (BMI) was $17 \mathrm{~kg} / \mathrm{m}^{2}$ at the admission time. The significant observation was a mobile mass $(2 \times 1 \mathrm{~cm})$ with soft consistency behind the left sternocleidomastoid in left cervical, axillary and inguinal lymph nodes; no redness or warmth was reported in the mass.

The spleen was detected about $5 \mathrm{~cm}$ below the rim. Ear, nose, and throat checkups showed normal results and the patient had no complaints of abdominal pain, lower limb pain, or diarrhea. In addition, no facial or body skin rash was reported and other findings were normal. In para-clinical evaluations, the results of chest radiograph, chest CT scan, electrocardiogram and echocardiography were normal. Moreover, in pelvic and abdominal ultrasounds, no abnormalities were observed except for the enlargement of the spleen. In the patient's initial blood test, white blood cell (WBC) count of 2000 cells $/ \mathrm{mm}^{3}$ and the presence of $1 \%$ atypical lymphocytes with an increase

Copyright (C) 2015, Ahvaz Jundishapur University of Medical Sciences. This is an open-access article distributed under the terms of the Creative Commons Attribution-NonCommercial 4.0 International License (http://creativecommons.org/licenses/by-nc/4.0/) which permits copy and redistribute the material just in noncommercial usages, provided the original work is properly cited. 
Erfanian Taghvaei MR et al.

in immature neutrophils (bands), hemoglobin of $11.3 \mathrm{~g} /$ $\mathrm{dL}$ and a mild thrombocytopenia, as well as high alkaline phosphatase, high C-reactive protein, and an erythrocyte sedimentation rate (ESR) of 48 were reported. Blood tests and examinations of urine and stool samples were all negative. The results of sputum acid-fast bacilli culture and antinuclear antibody test were negative, as well. Similarly, the results of autoimmune screening, as well as toxoplasma and cytomegalovirus tests were negative.

After 20 days, the patient was examined by neck lymph node biopsy. Macroscopic examination of the lymph node capsule showed a creamy grey-colored mass with soft consistency, homogeneity, and a dimension of $0.5 \times$ $1 \times 2 \mathrm{~cm}$. In the microscopic study (at low magnification), partial effacement of the lymph node structure and pale patchy infiltrates were reported. The number of small lymphocytes was fewer in the lesion. At the center of the lesion, an extensive coagulative necrosis composed of cellular debris and free of neutrophilic or eosinophilic infiltrates was observed (Figures 1 and 2).

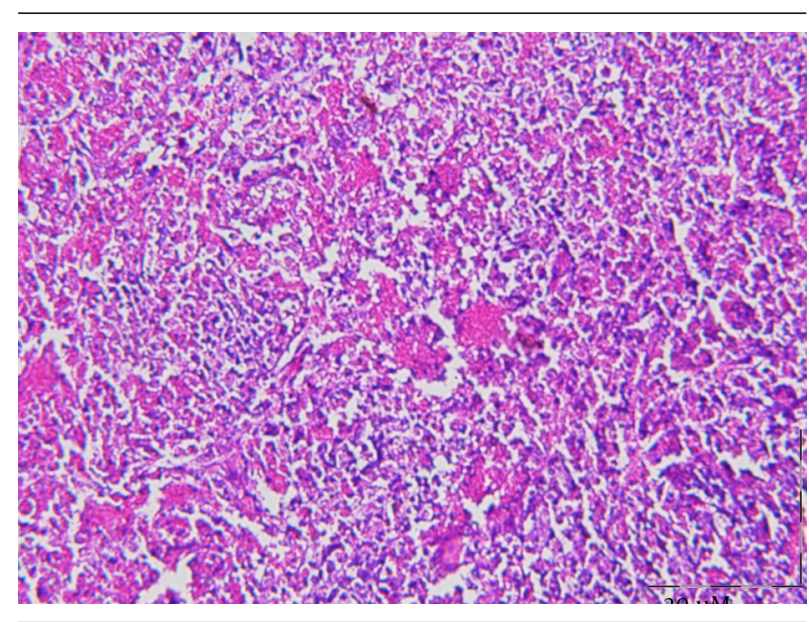

Figure 1. A Staining, Irregular Area Consisting of Minimal Necrosis, Surrounded by a Wide Zone of Immunoblasts and Histiocytes $(\mathrm{H}$ and E, $\times 20$ )

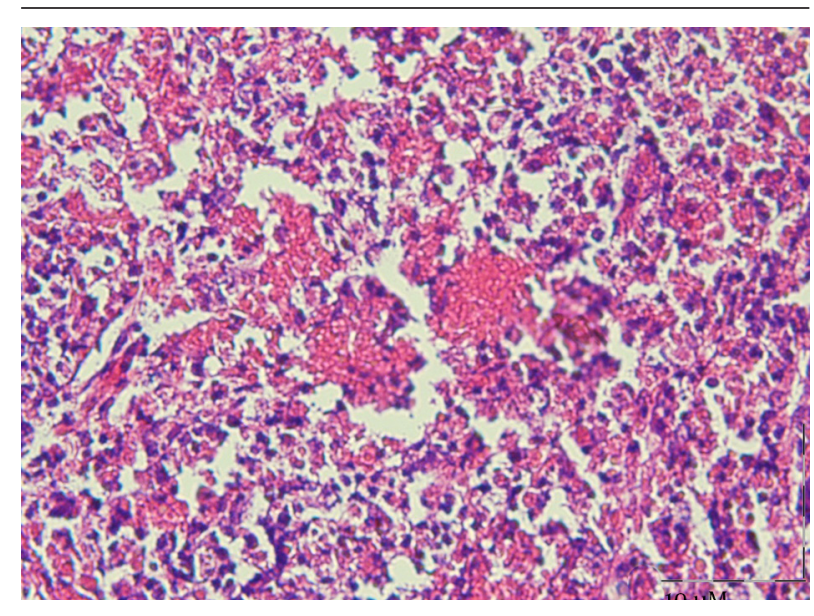

Figure 2. Excisional Biopsy of the Cervical Lymph Node Showing the Necrotic Area Devoid of Polymorphonuclear Leukocytes (H and E, × 40.)
Finally, the Kikuchi's disease was diagnosed based on the findings. Considering the improved symptoms of the patient after two weeks of hospitalization, she was discharged from the hospital in good general health and received anti-inflammatory medications. The patient was followed up one week after the discharge. Given the improvements in her symptoms, the medications were gradually discontinued and she started to be followed up on a monthly basis. During the 8-year follow-up of the patient, except for the mean sublingual temperature of $37.8^{\circ} \mathrm{C}$ which was reported once or twice a year (continuing for a couple of days), no other significant findings were reported. The patient was in good general health during the follow-up and her examination results were normal.

Blood cell count and ESR were within the normal range. The patient had two healthy pregnancies in 2010 and 2012 and was in a good general condition during her pregnancies and lactation. In August 2014, the patient was referred to the hospital with high fever, night sweats, weight loss and anorexia $\left(\mathrm{BMI}=16 \mathrm{~kg} / \mathrm{m}^{2}\right)$. After a one-month delay, painful generalized lymphadenopathy in left cervical, axillary and inguinal lymph nodes with soft consistency and mobility was reported; however, no redness or warmth was reported in the mass. In the second blood test, neither leukopenia nor high ESR was observed. The results of chest radiography as well as those of abdominal and pelvic ultrasounds were normal. Considering the patient's unwillingness to undergo repeated biopsy, she underwent a corticosteroid regimen. After three months of using prednisone, the patient's fever subsided and her lymphadenopathy was impalpable. Overall, the patient was in good general health.

\section{Discussion}

KFD, also known as HNL, is a self-limiting condition, causing lymphadenopathy. This disease is most commonly observed in adults younger than 40 years of age, with a female predominance in previous studies (3). Herein, we presented a case of a 32-year-old female with lymphadenopathy.

The most common clinical manifestation of KFD is cervical lymphadenopathy, followed by fever. Other less commonly reported presentations include leukopenia, atypical lymphocytes on peripheral smear, liver dysfunction, bone marrow involvement, fatigue, hepatosplenomegaly, and skin rash (2). Although the disease is characterized by regional lymphadenopathy, few patients show generalized lymphadenopathy. In the present case, there was simultaneous involvement of cervical lymph nodes with axillary and inguinal lymph nodes together with uncommon symptoms including leukopenia, severe weight loss and splenomegaly, indicating a generalized lymphoma which often leads to a misdiagnosis.

A definite diagnosis of KFD may be made only via histopathological analysis by open lymph node biopsy (1). 
There is no specific treatment for KFD, although in severe cases, the use of corticosteroids has been recommended for the prevention of fatal complications (1). The signs and symptoms of KFD usually resolve after several months (1, 2). In a review of cases with KFD by Kucukardali et al., the reported overall mortality due to KFD was estimated $2.1 \%$ (6). In addition, a low recurrence rate (3 - 4\%) has been reported in previous studies $(1,2)$. Patients with recurrent episodes are more likely to present fever, cough, and fatigue, along with frequent extranodal involvement in the initial presentation (7). In comparison with previous studies, the recurrence rate was remarkably higher in the present study. Our patient did not have a benign course. Her disease was complicated by the return of symptoms eight years after the initial diagnosis, with symptoms such as fever, fatigue, severe weight loss and generalized lymphadenopathy. In the present case, the use of corticosteroids suppressed the symptoms.

A definite diagnosis of KFD is made by tissue biopsy, particularly whole lymph node biopsy $(4,5)$. In the present case, the diagnosis of HNL was confirmed according to the results from the pathological slices; also, there is a chance that more severe cases get included with pathological confirmation, while mild cases get excluded due to follow-up loss. Although the etiology of KFD recurrence is unknown, certain viral infections including epstein-barr (EB) virus, parvovirus B19 and human herpesvirus-8 have been hypothesized to be among the triggers for KFD relapse (8). As Stephan et al. indicated, the recurrence of HNL is associated with the persistence of EB virus infections (9). In addition, Atarashi et al., reported a case of recurrent HNL in a human T-lymphotropic virus type I carrier. In the present case, infectious etiologies including EB virus, cytomegalovirus and HIV were all negative (10). It is unknown whether other viral infections were associated with HNL in the present case.

As evidence suggests, KFD may be a precursor for systemic lupus erythematosus (SLE), as both conditions have concurrent, coexisting patterns in patients (11). Moreover, an association between recurrent HNL and autoimmune diseases has been reported. Cheng et al., described the clinical manifestations and outcomes of 195 patients diagnosed with HNL. In the mentioned study, a total of 14 out of 96 patients (14.6\%) had recurrent HNL and five of them developed autoimmune diseases such as SLE (12). It seems that individuals with HNL are more susceptible to SLE; thus, they should be routinely screened for this disorder (2). With the results of the present study, since KFD is spontaneous, its recovery does not require any specific treatments. It is important to differentiate KFD from other conditions; in this case, it can inhibit adverse drug reactions in patients and prevent empirical treatments, especially with antibiotics that impose financial burdens on families.

In conclusion, although KFD is an uncommon condi- tion, it should be featured in the list of differential diagnoses of tender lymphadenopathy, especially lymphadenopathy localized to the cervical region. Our patient exhibited generalized lymphadenopathy which was uncommon. However, although the disease takes a selflimiting clinical course in most cases, we reported a case of KFD with a prolonged relapse of eight years. Full recovery with a good response to corticosteroid regimen was achieved after the recurrence; therefore, considering the recurrence of KFD, long-term follow-up of patients with KFD is necessary.

\section{Authors' Contributions}

Majid Reza Erfanian Taghvaei developed the original idea and the protocol. Maryam Mirzaie abstracted the data and wrote the manuscript. Ali Parsa contributed to the development of the protocol, abstracted the data, and prepared the manuscript. Taghi Ghiasi Moghadam wrote the context and prepared the figures of the pathology section of the manuscript. Maryam Mirzaie supervised the study.

\section{References}

1. Hutchinson CB, Wang E. Kikuchi-Fujimoto disease. Arch Pathol Lab Med. 2010;134(2):289-93.

2. Rezai K, Kuchipudi S, Chundi V, Ariga R, Loew J, Sha BE. KikuchiFujimoto disease: hydroxychloroquine as a treatment. Clin Infect Dis. 2004;39(12):e124-6.

3. Tsang WY, Chan JK, Ng CS. Kikuchi's lymphadenitis. A morphologic analysis of 75 cases with special reference to unusual features. Am J Surg Pathol.1994;18(3):219-31.

4. Pileri S, Kikuchi M, Helbron D, Lennert K. Histiocytic necrotizing lymphadenitis without granulocytic infiltration. Virchows Arch A Pathol Anat Histol. 1982;395(3):257-71.

5. Kuo TT. Cutaneous manifestation of Kikuchi's histiocytic necrotizing lymphadenitis. Am J Surg Pathol.1990;14(9):872-6.

6. Kucukardali Y, Solmazgul E, Kunter E, Oncul O, Yildirim S, Kaplan M. Kikuchi-Fujimoto Disease: analysis of 244 cases. Clin Rheumatol. 2007;26(1):50-4.

7. Song JY, Lee J, Park DW, Sohn JW, Suh SI, Kim IS, et al. Clinical outcome and predictive factors of recurrence among patients with Kikuchi's disease. Int J Infect Dis. 2009;13(3):322-6.

8. Lee HY, Huang YC, Lin TY, Huang JL, Yang CP, Hsueh T, et al. Primary Epstein-Barr virus infection associated with Kikuchi's disease and hemophagocytic lymphohistiocytosis: a case report and review of the literature. J Microbiol Immunol Infect. 2010;43(3):253-7.

9. Stephan JL, Jeannoel P, Chanoz J, Gentil-Perret A. Epstein-Barr virus-associated Kikuchi disease in two children.J Pediatr Hematol Oncol. 2001;23(4):240-3.

10. Atarashi K, Yoshimura N, Nodera H, Tsukimoto K, Beppu H, Kanayama M. Recurrent histiocytic necrotizing lymphadenitis (Kikuchi's disease) in an human T lymphotropic virus type I carrier. Intern Med. 1996;35(10):821-5.

11. Santana A, Lessa B, Galrao L, Lima I, Santiago M. Kikuchi-Fujimoto's disease associated with systemic lupus erythematosus: case report and review of the literature. Clin Rheumatol. 2005;24(1):60-3.

12. Cheng CY, Sheng WH, Lo YC, Chung CS, Chen YC, Chang SC. Clinical presentations, laboratory results and outcomes of patients with Kikuchi's disease: emphasis on the association between recurrent Kikuchi's disease and autoimmune diseases. J Microbiol Immunol Infect. 2010;43(5):366-71. 